\title{
A New Retinoid-Like Compound That Activates Peroxisome Proliferator- Activated Receptors and Lowers Blood Glucose in Diabetic Mice
}

\author{
Tuo Deng,${ }^{a, b}$ Song Shan, ${ }^{b}$ Zhi-Bin Li, ${ }^{b}$ Zhong-Wen Wu, ${ }^{b}$ Chen-Zhong Liao, ${ }^{b}$ Ben Ko, ${ }^{c}$ \\ Xian-Ping Lu, ${ }^{b}$ Jing CHENG, ${ }^{a, b}$ and Zhi-Qiang Ning ${ }^{*}, b$ \\ ${ }^{a}$ Department of Biological Science and Biotechnology, Tsinghua University; Beijing: ${ }^{b}$ Chipscreen Biosciences Ltd., \\ Research Institute of Tsinghua University; Shenzhen: and ${ }^{c}$ Institute of Molecular Biology, University of Hong Kong; \\ Hongkong, China. Received January 24, 2005; accepted March 24, 2005
}

Retinoid X receptor (RXR) forms heterodimers with peroxisome proliferator-activated receptors (PPARs, with subtypes of $\alpha, \delta$ and $\gamma$ ), and the heterodimers can be activated by either an RXR or a PPAR subtype-specific ligand. Based on the chemical structure of the RXR natural ligand, 9-cis-retinoic acid (9-cis-RA), we designed and synthesized a retinoid-like compound, CS018. In vitro characterizations by cell-based reporter gene assays indicated that CS018 activated RXR homodimers and the heterodimers of RXR with PPARs, but not with farnesoid X-activated receptor (FXR) and liver X-activated receptor (LXR). Furthermore, RT-PCR results showed that CS018 induced the expression of the PPAR $\gamma$ target genes, CD36 and lipoprotein lipase (LPL). In vivo studies on the diabetic $d b / d b$ mice demonstrated that CS018 dramatically lowered the animal blood glucose levels. CS018 thus may represent a new retinoid-like compound that activates RXR/PPARs and has potential therapeutic applications in type $\mathbf{2}$ diabetes and other metabolic diseases.

Key words synthetic compound; peroxisome proliferator-activated receptor; retinoid X receptor; type 2 diabetes

New insights into the members of ligand-activated nuclear receptors called peroxisome proliferator-activated receptors (PPARs) have opened up the possibilities for treatment of many metabolic diseases including type 2 diabetes. ${ }^{1)}$ PPARs have three subtypes, i.e. $\operatorname{PPAR} \alpha, \operatorname{PPAR} \delta$, and $\operatorname{PPAR} \gamma$. PPARs modulate expression of genes involved in the transport and metabolism of glucose and lipids. ${ }^{2)}$ PPAR $\alpha$ is mainly expressed in tissues such as liver, which is activated by a class of ligands named fibrates that are known to have triglyceride- and cholesterol-lowering activity. ${ }^{3)}$ PPAR $\delta$ regulates fat metabolism and may serve as a potential target in treatment of obesity and its associated disorders. ${ }^{4)}$ Activation of PPAR $\gamma$ mediates adipocyte differentiation and storage of free fatty acids primarily into adipose tissue, and the PPAR $\gamma$ agonists have been widely used in treatment of type 2 diabetes. ${ }^{5}$

All three PPARs form heterodimers with another nuclear receptor, retinoid X receptor (RXR). RXR/PPARs belong to permissive RXR heterodimers which can be activated by either an RXR or a PPAR subtype-specific ligand. $\left.{ }^{6}\right)$ Synthetic compounds binding to either RXR/RXR homodimers or $\mathrm{RXR} / \mathrm{PPAR} \gamma$ heterodimers have been shown to relieve insulin resistance in various animal models of type 2 diabetes. ${ }^{7}$ Those compounds include the RXR/RXR antagonist LG100754, ${ }^{8,9)}$ and the well known RXR/PPAR $\gamma$ agonists thiazolidinediones (TZDs, for example, rosiglitazone). ${ }^{10)}$ Thus, synthesis and characterization of novel compounds acting on either RXR/RXR and/or RXR/PPARs will be a reasonable strategy to discover the potential drug leads for the treatment of metabolic diseases.

Based on the chemical structure of the RXR natural ligand, 9-cis-retinoic acid (9-cis-RA), we designed and synthesized a retinoid-like compound, CS018. To evaluate whether CS018 could be further developed as a drug lead for the treatment of type 2 diabetes, we have characterized the properties of CS018 in activation of RXR-related receptors in vitro, and observed its blood glucose-lowering effect in vivo, as reported in the present study.

\section{MATERIALS AND METHODS}

Chemistry of CS018 CS018 [Ethyl(E,E,E)-7-(2-npropoxy-5,5,8,8-tetramethyl-5,6,7,8-tetrahydro-naphthalen3-yl)-6-fluoro-3-methylocta-2,4,6-trienoate, with a molecular weight of 414.57] was synthesized with the starting materials 5,5,8,8-tetramethyl-5,6,7,8-tetrahydro- $b$-naphthol, triethyl $b$ fluoro- $b$-phosphonoacetate and triethyl 3-methyl-4-phosphonocrotonate. There were 8 steps for the synthesis, and the total yield was $8.4 \%$. The chemical structure of CS018 is shown in Fig. 1.

Reagents and Plasmids Chenodeoxycholic acid (CDCA), 22(R)hydroxycholesterol (22(R)OHC), 2-Bromohexadecanoic acid (2-Bro) and 9-cis-RA were purchased from Sigma (St. Louis, Mo, U.S.A.). Wy-14643 was purchased from Biomol (Plymouth, PA, U.S.A.). Rosiglitazone was synthesized at Chipscreen Biosciences Ltd. (Shenzhen, China). The human cDNAs for $\operatorname{RXR} \alpha, \operatorname{PPAR} \gamma, \operatorname{LXR} \alpha$, and FXR were obtained by RT-PCR amplification from mRNA isolated from liver or adipose tissues. The amplified cDNA inserts were cloned into pcDNA3.1 (Invitrogen, Carlsbad, CA, U.S.A.) and sequenced to confirm the right reading frames. The PPAR $\delta$ expression plasmid was a kind gift of Dr. H. Jiang (Shanghai Institute of Matera Medica, China). pSG5-hPPAR $\alpha$, an expression vector for human PPAR $\alpha$, was kindly provided by Dr. P. Gervois (Institut Pasteur de Lille, France). Luciferase reporter plasmids, ACOX PPRE (for $\operatorname{PPAR} \gamma$ ) and $\mathrm{pHD}(\mathrm{X} 3) l u c$ (for $\operatorname{PPAR} \alpha$ ), were kind gifts of Drs. T. M. McIntyre (University of Utah) and R. A. Rachubinski (University of Alberta, Canada), respectively. Lu-

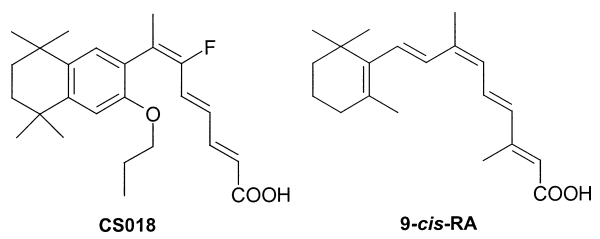

Fig. 1. Chemical Structures of CS018 and 9-cis-Retinoic Acid (9-cis-RA) 
ciferase reporter plasmids, PPRDE-luc (for PPAR $\delta$ ), RXRE$l u c$ (for RXR), FXRE-luc (for FXR) and LXRE-luc (for LXR), were constructed by insertion of the annealed oligonucleotides into the upstream of the luciferase gene in pGL3-Promoter vector (Promega, Madison, WI, U.S.A.). The annealed oligonucleotides containing the corresponding response elements in each reporter plasmid are the following: $5^{\prime}$-gatctaggtcaaaggtcaaatctaggtcaaaggtcaaatctaggtcaaaggtcaaatctaggtcaaaggtcaaatcg-3' in PPRDE-luc, 5' -gatctaggtcagaggtcagagagctaggtcagaggtcaga gagctaggtcagaggtcagagag- $3^{\prime}$ in RXRE-luc, 5'-tcgagaactgagggtcagtgacccaagtgctcgagaactgagggtcagtgacccaagtgctcgagaactgagggtcagtgacccaagtgc- $3^{\prime}$ in FXRE-luc, and $5^{\prime}$-tcgagctttggtcactcaagttcaagttactcgagctttggtcactcaagttcaagttactcgagctttggtcactcaagttcaagttac- $3^{\prime}$ in LXREluc. pCMV $\beta$ Gal was purchased from Clontech (Palo Alto, CA, U.S.A.).

Reporter Gene Assay The CV-1 cell line was obtained from the American Type Culture Collection (ATCC). CV-1 cells were cultured in DMEM with $10 \%$ heat-inactivated fetal bovine serum (FBS, Invitrogen, Grand Island, NY, U.S.A.) in a humidified $5 \% \mathrm{CO}_{2}$ atmosphere at $37^{\circ} \mathrm{C}$. Cells were seeded in 96-well plates the day before transfection to give a confluence of $50-80 \%$ at transfection. A total of $60 \mathrm{ng}$ of DNA containing $10 \mathrm{ng}$ of $\operatorname{hRXR} \alpha, 10 \mathrm{ng}$ of pCMV $\beta G a l, 10 \mathrm{ng}$ of a designated nuclear receptor expression vector and $30 \mathrm{ng}$ of the corresponding reporter plasmid, was transfected per well using the FuGene6 transfection reagent (Roche Molecular Biochemicals, Indianapolis, IN, U.S.A.) according to the manufacturers instructions. Twentyfour hours after transfection, the culture media were changed to $10 \%$ charcoal-stripped FBS in DMEM containing the indicated compounds dissolved in DMSO. The final concentration of DMSO in culture medium $(200 \mu \mathrm{l})$ was $0.1 \%$. Cells were incubated with the indicated compounds for $24 \mathrm{~h}$, then collected with Cell Culture Lysis buffer (Promega). Luciferase activity was monitored using the luciferase assay kit (Promega) according to the manufacturers instructions. Light emission was read on an Ascent Fluoroskan FL reader (Thermo Labsystems, Finland). $\beta$-Galactosidase assays were performed to normalize the transfection conditions. To measure the $\beta$-galactosidase activity by a kit from Promega, $50 \mu \mathrm{l}$ of supernatant from each transfection lysate was transferred to a new microplate, and read at the light wave of $405 \mathrm{~nm}$ in a microplate reader (Bio-tek Instruments Inc., Winooski, VT, U.S.A.).

Gene Induction by RT-PCR Analysis Two-day post confluent 3T3-L1 cells (mouse preadipocyte cells provided by ATCC) were treated with different compounds for $48 \mathrm{~h}$. Total RNA was extracted by the TRIzol reagent (Invitrogen). The first strand cDNA synthesis containing $2 \mu \mathrm{g}$ of total RNA was primed with oligo(dT) and used SuperScript II reverse transcriptase (Invitrogen) according to the manufacturers instructions. Information about primers and cycles for
PCR was given in Table 1. The 36B4 gene was used as an internal standard for the PCR analysis.

In Vivo Study on $\boldsymbol{d} \boldsymbol{b} / \boldsymbol{d} \boldsymbol{b}$ Mice The male $d b / d b$ mice (BKS. Cg- $m+/+$ Lepr $\left.^{d b}\right)$ at the age of 6 weeks were purchased from The Jackson Laboratory (Bar Harbor, ME, U.S.A.). Blood glucose levels were monitored by the Blood Glucose Test Strips (Bayer Diagnostics Division, Tarrytown, NY, U.S.A.) every 3-d upon the animal arrival. Experiments were carried out from the 9-week old animals, whose average blood glucose concentrations reached about $13 \mathrm{~mm}$ at the time. $d b / d b$ mice were orally administered once daily with the vehicle, CS018 $(80 \mathrm{mg} / \mathrm{kg})$ or rosiglitazone $(5 \mathrm{mg} / \mathrm{kg})$ for contiguous 9 days, and the blood glucose concentrations were monitored every three days after 3-h fasting.

Statistical Analysis Results are expressed as mean \pm S.D. The significance of difference was analyzed by Student's $t$-test.

\section{RESULTS}

Effect of CS018 on RXR Homodimers CS018 has structural similarities with the RXR natural ligand, 9-cis-RA (Fig. 1). To functionally test whether CS018 had any effect on the transactivating activity of RXR homodimers, CV-1 cells were transfected with an RXR expression vector, together with a luciferase reporter construct having 3 copies of consensus RXR response element upstream of the minimal promoter, and the transactivation of the luciferase gene was determined. As shown in Fig. 2, CS018 had a dose-dependent $(0.1-10 \mu \mathrm{M})$ activation in RXR/RXR transactivating activity, though weaker than that of 9-cis-RA (about 18\% at $0.1 \mu \mathrm{M}$ concentration). When the transactivation assays were conducted in the presence of a fixed concentration of 9-cisRA at $0.1 \mu \mathrm{M}, \mathrm{CS} 018$ produced inhibitory effect on the 9-cisRA-induced RXR/RXR activation (Fig. 2). The results indicate that CS018 by itself is a weak agonist of RXR, but an antagonist in the presence of the natural ligand of the recep-

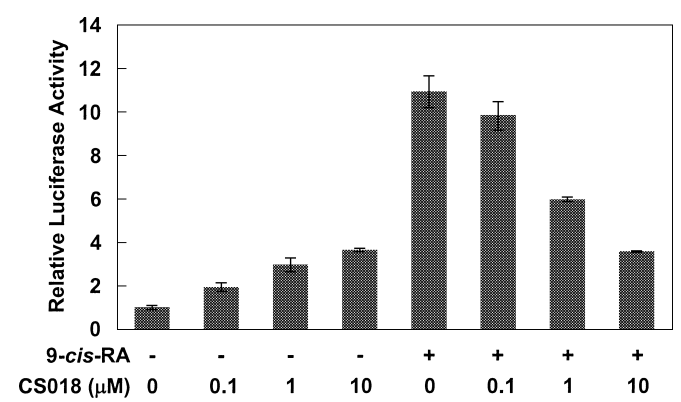

Fig. 2. Dual Actions of CS018 on RXR/RXR Activation

CV-1 cells were transfected with the RXR expression vector together with the RXRE-luc reporter construct. Twenty-four hours after transfection, cells were incubated with increasing concentrations of CS018 in the presence or absence of 9-cis-RA $(0.1 \mu \mathrm{M})$ for $24 \mathrm{~h}$, and luciferase activity was determined. The results are presented as mean \pm S.D. from 3 independent experiments, with triplicates in each experiment.

Table 1. Primer Sets and the Number of Cycles Used for the Semiquantitative PCR

\begin{tabular}{lllc}
\hline \hline Gene & \multicolumn{1}{c}{ Sense primer $\left(5^{\prime}\right.$ to $\left.3^{\prime}\right)$} & Antisense primer $\left(5^{\prime}\right.$ to $\left.3^{\prime}\right)$ & The number of cycles \\
\hline CD36 & ACAGACGCAGCCTCCTTTCC & GTCCCAGTCTCATTTAGCCACAG & 21 \\
LPL & CCCCAGTCGCCTTTCTCCTGAT & CTCTTGGCTCTGACCTTGTTGAT & 18 \\
36B4 & CCGTGGTGCTGATGGGCAAGAA & CCCAAAGCCTGGAAGAAGGA & 22 \\
\hline
\end{tabular}



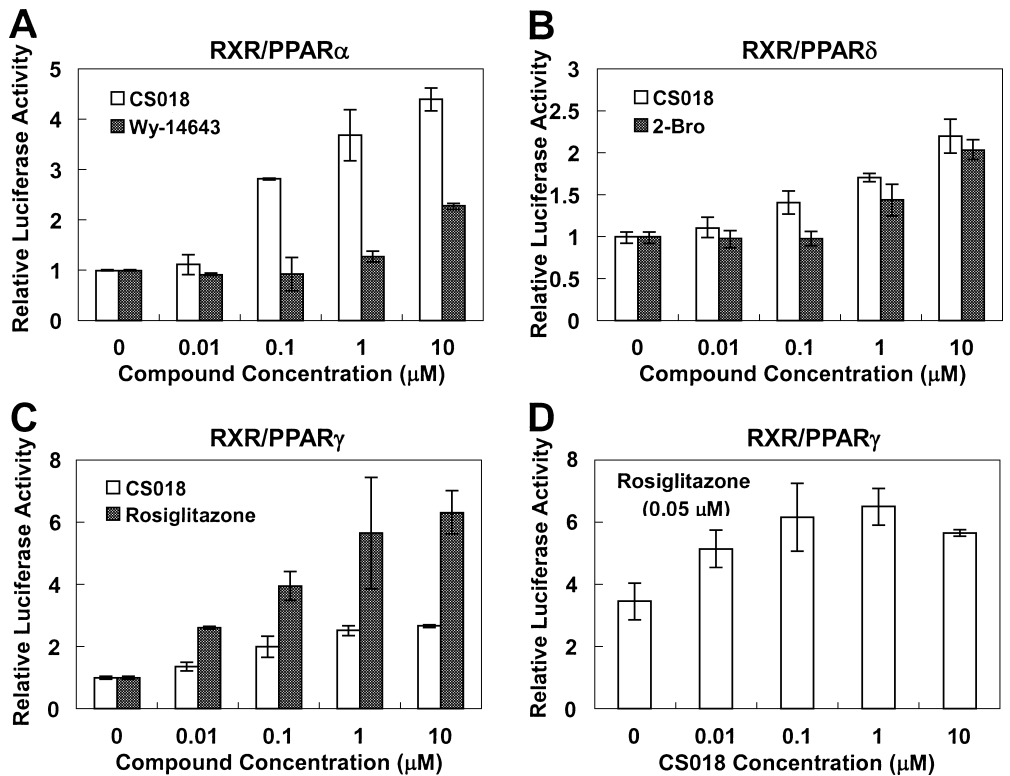

Fig. 3. Activation of RXR/PPARs by CS018

CV-1 cells were co-transfected with RXR and the indicated subtype of PPAR expression vectors together with a reporter gene construct having the corresponding PPAR response element. Twenty-four hours after transfection, cells were incubated with the indicated concentrations of CS018 alone (A, B, C), or combined with $0.05 \mu \mathrm{M}$ of rosiglitazone (D) for $24 \mathrm{~h}$, and luciferase activity was determined. For comparison of the receptor activation by CS018 with the known agonists, luciferase activity induced by equimolar concentrations of Wy-14643 (A), 2-bromohexadecanoic acid (B), or rosiglitazone (C), were also included in the corresponding figures. The results are presented as mean \pm S.D. from 3 independent experiments, with triplicates in each experiment.

tor.

Effect of CS018 on RXR/PPARs To functionally test whether CS018 activated RXR-formed heterodimers with subtypes of individual PPARs, CV-1 cells were transfected with a luciferase reporter construct having the designated PPAR binding element in it, together with a vector expressing RXR plus a vector expressing either $\operatorname{PPAR} \alpha, \operatorname{PPAR} \delta$, or $\operatorname{PPAR} \gamma$, and CS018-mediated transactivation in the luciferase gene through the individual subtypes of PPARs was determined. As the results presented in Fig. 3, CS018 showed remarkable activation in all those RXR-formed PPAR heterodimers. Compared with the known PPAR agonists at the equimolar concentrations, CS018 had a stronger activating effect on PPAR $\alpha$ than Wy-14643 (Fig. 3A), roughly equivalent activating effect on $\operatorname{PPAR} \delta$ with 2-bromohexadecanoic acid (Fig. 3B), and weaker activating effect on PPAR $\gamma$ than rosiglitazone (Fig. 3C). Activating effect of CS018 on PPAR $\gamma$ in the presence of rosiglitazone was also investigated. Interestingly, unlike the inhibitory effect on the ligand-induced activation of RXR/RXR homodimers, CS018 showed no inhibitory, but rather synergistic effect, on the agonist-induced activation of RXR/PPAR $\gamma$ heterodimers (Fig. 3D).

To investigate whether CS018 could induce any PPAR $\gamma$ target genes, the mouse preadipocyte cell line, 3T3-L1, which has the endogenous RXR/PPAR $\gamma$ expression, was treated with CS018 and the expression of the known PPAR $\gamma$ target genes, CD36 ${ }^{11)}$ and LPL, ${ }^{12)}$ was evaluated by RT-PCR. As shown in Fig. 4, both $\mathrm{CS} 018$ and rosiglitazone induced CD36 and LPL expression. Consistent with the reporter gene assays, CS018 showed weaker induction of PPAR $\gamma$ target genes than rosiglitazone (Fig. 4).

Effect of CS018 on RXR/FXR and RXR/LXR In the similar reporter gene assay system, functional effect of CS018 on transactivating activity of other RXR heterodimers, FXR and LXR, was also determined in CV-1

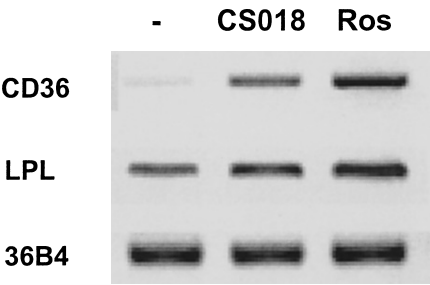

Fig. 4. Induction of the PPAR $\gamma$ Target Genes by CS018

The two-day post confluent 3T3-L1 cells were treated with either CS018 $(5 \mu \mathrm{M})$ or rosiglitazone $(1 \mu \mathrm{M})$ for $48 \mathrm{~h}$. Total RNA was isolated, and the semi-quantitative RTPCR was carried out. 36B4 was used as an internal control.

cells. As shown in Fig. 5, CS018 by itself activated neither RXR/FXR, nor RXR/LXR, at the concentration $(5 \mu \mathrm{M})$ tested. However, CS018 demonstrated inhibitory effect on the agonist-induced activation of the two RXR heterodimers when CV-1 cells were co-treated with CS018 and the agonists (CDCA for RXR/FXR, and 22(R)OHC for RXR/LXR, respectively). The results indicate that $\mathrm{CS} 018$ has more selective activity in RXR/RXR and RXR/PPAR receptors, providing a scientific basis for the further investigation of CS018 as an agent for anti-diabetes or other metabolic diseases.

Effect of CS018 on Blood Glucose Levels in $d b / d b$ Mice To conceptually test whether CS018 had any influence on the blood glucose levels in vivo, $d b / d b$ mice at 9-weeks of age were orally administered with a single dose $(80 \mathrm{mg} / \mathrm{kg})$ of CS018 for 9 contiguous days, and the blood glucose concentrations were determined at the indicated days before and after compound treatment. As shown in Fig. 6, treatment of $\mathrm{db} / \mathrm{db}$ mice with CS018 lowered blood glucose rapidly and persistently, with similar kinetics and intensity compared with rosiglitazone at the dose of $5 \mathrm{mg} / \mathrm{kg}$. 


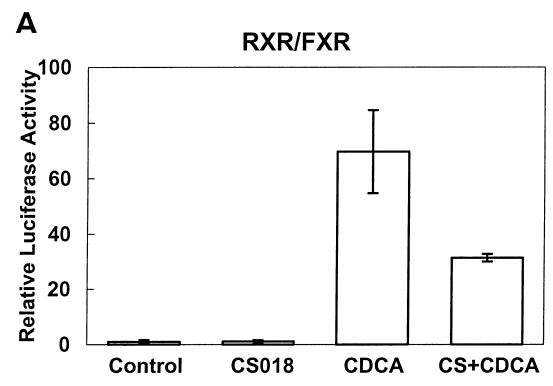

B

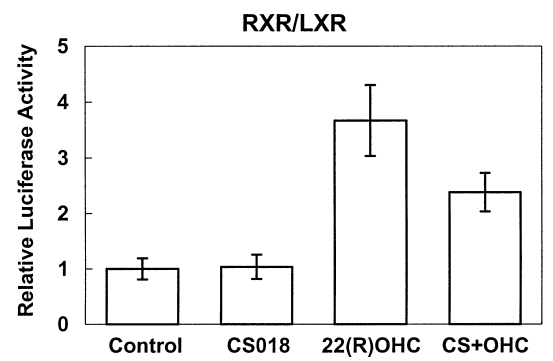

Fig. 5. CS018 Does Not Activate RXR/FXR and RXR/LXR

CV-1 cells were co-transfected with the RXR and FXR expression vectors together with the FXRE-luc reporter plasmid (A), or RXR and LXR expression vectors together with the LXRE-luc reporter plasmid (B). Twenty-four hours after transfection, cells were treated with the indicated compounds or their combinations for $24 \mathrm{~h}$, and luciferase activity was determined. The results are presented as mean \pm S.D. from 3 independent experiments, with triplicates in each experiment. Compound concentrations used: CS018 $(5 \mu \mathrm{M}), \operatorname{CDCA}(50 \mu \mathrm{M})$, and $22(R) \mathrm{OHC}(10 \mu \mathrm{M})$. Control only contained DMSO $(0.1 \%)$.

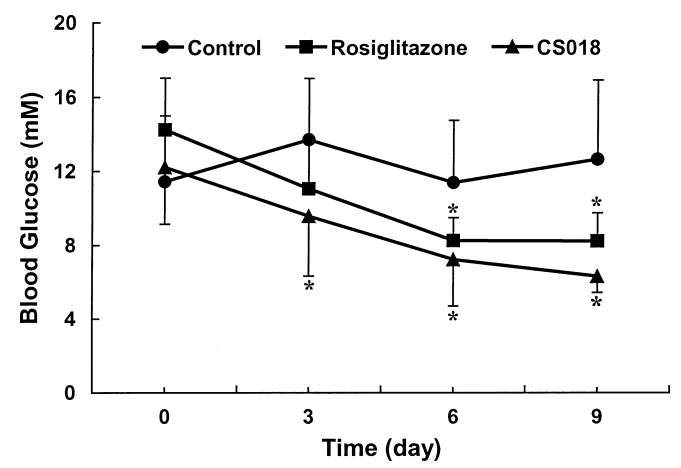

Fig. 6. CS018 Lowers Blood Glucose Levels in $d b / d b$ Mice

Nine-week $d b / d b$ mice were treated with $\mathrm{CS} 018(80 \mathrm{mg} / \mathrm{kg})$ or rosiglitazone $(5 \mathrm{mg} / \mathrm{kg})$ daily for 9 contiguous days. Blood glucose levels were monitored every three days. Seven mice were used in each group. $* p<0.05 v s$. control.

\section{DISCUSSION}

PPARs are nuclear hormone receptors that bind peroxisome proliferators and control the size and number of peroxisomes produced by cells. PPARs mediate a variety of biological processes, and are involved in the development of several chronic diseases, including diabetes, obesity, and atherosclerosis. In fact, PPAR agonists have been used as anti-diabetes and triglyceride-lowering drugs. ${ }^{13)}$

Since RXR/PPARs belong to RXR permissive heterodimers, compounds bind to and act on RXR may also cause PPAR activation, as exemplified by compounds LG 100268 and LG100754. ${ }^{8,9)}$ Based on this principle as well as the chemical structure of the RXR natural ligand 9-cis-RA, we synthesized a new retinoid-like compound, CS018.

In vitro characterizations by the cell-based reporter gene system have functionally demonstrated that (1) CS018 by it- self is a weak agonist of the RXR homodimers, but it is an antagonist in the presence of the RXR natural ligand; (2) CS018 is a pan agonist of the RXR-formed heterodimers with $\operatorname{PPAR} \alpha, \delta$, and $\gamma$, and synergizes $\operatorname{PPAR} \gamma$ activation by rosiglitazone; and (3) CS018 does not activate other RXR heterodimers tested, including RXR/FXR and RXR/LXR, but antagonizes the activation of those receptors by their agonists. The mechanisms for the favorable activation of the RXR/PPAR heterodimers by CS018 are not known yet. Different RXR ligands may initiate different cascades of interactions between receptor dimmers, cofactors, and members of the transcription machinery. ${ }^{8)}$ Thus, upon the receptor binding, CS018 might act on a selective pathway that favorably enhances the transcriptional activity by RXR-formed heterodimers with PPARs.

Compared with the known synthetic RXR acting compounds, LG100268 and LG100754, CS018 shows more similarities with the compound LG100754 in terms of the activating selectivity in the receptor dimmers formed by RXR. While LG100268 is a pan-agonist to all RXR-formed homodimers and heterodimers, ${ }^{8,9)}$ CS018 and LG100754 show more selectivity in activating RXR/PPAR heterodimers. However, minor differences of CS018 and LG100754 are also noticed from the reference reports as well as our own observations. For instance, LG100754 is shown to be an antagonist of RXR homodimers, ${ }^{8)}$ whereas CS018 is a weak agonist of the homodimers, but an antagonist in the presence of the natural ligand of the receptor (Fig. 2). Nevertheless, the relative selectivity of CS018 in activation of RXR/PPAR heterodimers may be proved to be an advantage in development of the compound for potential therapeutic applications.

Activation of PPAR $\gamma$ by CS018 has been confirmed by gene expression analysis, in which CD36 and LPL in a mouse preadipocyte cell line are up-regulated by the compound. Both CD36 and LPL are well defined as the PPAR $\gamma$ target genes and are involved in the human insulin-resistance syndromes. ${ }^{14-17)}$ Results from the diabetic $d b / d b$ mice treated with CS018 have demonstrated that the compound dramatically lowers blood glucose levels in the animal, further indicating that CS018 may function as an insulin sensitizer by activating PPARs.

In conclusion, the presented results demonstrate that CS018 represents a new retinoid-like compound that activates RXR/PPARs and has potential therapeutic applications in type 2 diabetes and other metabolic diseases.

Acknowledment This study was partially funded by the National "863" Project of the People's Republic of China (No. 2002AA2Z3146).

\section{REFERENCES}

1) Moller D. E., Nature (London), 414, 821-827 (2001).

2) Desvergne B., Wahli W., Endocr Rev., 20, 649-688 (1999).

3) Duval C., Fruchart J., Staels B., Arch. Mal. Coeur. Vaiss., 97, 665672 (2004).

4) Gurnell M., Savage D. B., Chatterjee V. K., O'Rahilly S., J. Clin. Endocrinol. Metab., 88, 2412-2421 (2003).

5) Wang Y. X., Lee C. H., Tiep S., Yu R. T., Ham J., Kang H., Evans R. M., Cell, 113, 159-170 (2003).

6) Forman B. M., Umesono K., Chen J., Evans R. M., Cell, 81, 541-550 (1995).

7) Mukherjee R., Davies P. J., Crombie D. L., Bischoff E. D., Cesario R. 
M., Jow L., Hamann L. G., Boehm M. F., Mondon C. E., Nadzan A. M., Paterniti J. R., Jr., Heyman R. A., Nature (London), 386, 407410 (1997).

8) Lala D. S., Mukherjee R., Schulman I. G., Koch S. S., Dardashti L. J., Nadzan A. M., Croston G. E., Evans R. M., Heyman R. A., Nature (London), 383, 450-453 (1996).

9) Cesario R. M., Klausing K., Razzaghi H., Crombie D., Rungta D., Heyman R. A., Lala D. S., Mol. Endocrinol., 15, 1360-1369 (2001).

10) Lehmann J. M., Moore L. B., Smith-Oliver T. A., Wilkison W. O., Willson T. M., Kliewer S. A., J. Biol. Chem., 270, 12953-12956 (1995).

11) Feng J., Han J., Pearce S. F., Silverstein R. L., Gotto A. M., Jr., Hajjar D. P., Nicholson A. C., J. Lipid. Res., 41, 688-696 (2000).
12) Schoonjans K., Peinado-Onsurbe J., Lefebvre A. M., Heyman R. A., Briggs M., Deeb S., Staels B., Auwerx J., Embo. J., 15, 5336-5348 (1996).

13) Willson T. M., Brown P. J., Sternbach D. D., Henke B. R., J. Med. Chem., 43, 527-550 (2000).

14) Febbraio M., Abumrad N. A., Hajjar D. P., Sharma K., Cheng W., Pearce S. F. A., Silverstein R. L., J. Biol. Chem., 274, 19055-19062 (1999).

15) Aitman T., Glazier A., Wallace C., Cooper L., Norsworthy P., Wahid F., Nat. Genet., 21, 76-83 (1999).

16) Havel R., Gordon R.S. J., J. Clin. Invest., 39, 1777-1790 (1960).

17) Heizmann C., Kirchgessner T., Kwiterovich P., Ladias J., Derby C., Antonarakis S., Lusis A., Hum. Genet., 86, 578-584 (1991). 